\title{
Some eigenvalue results for perturbations of maximal monotone operators
}

In-Sook Kim and Jung-Hyun Bae

"Correspondence: iskim@skku.edu Department of Mathematics,

Sungkyunkwan University, Suwon, 440-746, Republic of Korea

\begin{abstract}
We study the solvability of a nonlinear eigenvalue problem for maximal monotone operators under a normalization observation. The investigation is based on degree theories for appropriate classes of operators, and a regularization method by the duality operator is used. Let $X$ be a real reflexive Banach space with its dual $X^{*}$ and $\Omega$ be a bounded open set in $X$. Suppose that $T: D(T) \subset X \rightarrow X^{*}$ is a maximal monotone operator and $C:(0, \infty) \times \bar{\Omega} \rightarrow X^{*}$ is a bounded demicontinuous operator satisfying condition $\left(S_{+}\right)$. Applying the Browder degree theory, we solve a nonlinear eigenvalue problem of the form $T x+C(\lambda, x)=0$. In the case where $T x+\lambda C x=0$, an eigenvalue result for generalized pseudomonotone densely defined perturbations is obtained by the Kartsatos-Skrypnik degree theory.

MSC: 47J10; 47H05; 47H14; 47H11
\end{abstract}

Keywords: eigenvalue; maximal monotone operator; perturbation; degree theory

\section{Introduction and preliminaries}

Eigenvalue theory is closely related to the problem of solving nonlinear equations which was initiated by Krasnosel'skii [1] for compact operators. Regarding maximal monotone operators, it has been extensively investigated by many researchers in various aspects, with applications to evolution equations and differential equations; see, e.g., [2-5]. The study was mostly based on degree theories for appropriate classes of operators and the usual method of regularization by means of the duality operator; see [6-10].

Let $X$ be a real reflexive Banach space with its dual $X^{*}$ and $\Omega$ be a bounded open set in $X$. Suppose that $T: D(T) \subset X \rightarrow X^{*}$ is a maximal monotone operator. We consider the nonlinear eigenvalue problem

$$
T x+\lambda C x=0,
$$

where $C: D(C) \subset X \rightarrow X^{*}$ is an operator. When the operator $C$ or the resolvents of the operator $T$ are compact, this problem was studied, for instance, by Guan-Kartsatos [11], Kartsatos [12], and Li-Huang [13], where the method is to use the Leray-Schauder degree theory. More generally, an implicit eigenvalue problem of the form

$$
T x+C(\lambda, x)=0
$$

was investigated in $[3,4]$, where $C:(0, \infty) \times \bar{\Omega} \rightarrow X^{*}$ is a bounded operator to be specified later. Kartsatos and Skrypnik [4] observed the above eigenvalue problem provided that the

(c) 2013 Kim and Bae; licensee Springer. This is an Open Access article distributed under the terms of the Creative Commons Attribution License (http://creativecommons.org/licenses/by/2.0), which permits unrestricted use, distribution, and reproduction in any medium, provided the original work is properly cited. 
following property $(\mathcal{P})$ is fulfilled: For $\varepsilon>0$, there exists a $\lambda>0$ such that the equation

$$
T x+C(\lambda, x)+\varepsilon J x=0
$$

has no solution in $D(T) \cap \Omega$, where $J$ denotes the duality operator. This property has a close relation to the use of topological degree in eigenvalue theory by the regularization method. It is shown in [3] that two conditions about the weak closure of a certain set consisting of normalized vectors and the asymptotic behavior of the operator $C$ at infinity of $\lambda$, called normalized conditions, are main ingredients in solving an eigenvalue problem. In this connection, we are now interested in finding eigenvectors under normalized conditions more concrete than property $(\mathcal{P})$.

The purpose of this paper is to establish the existence of solutions for the above eigenvalue problems under normalized conditions, motivated by the works of Kartsatos and Skrypnik [3, 4]. We first study implicit eigenvalue problem (IE), where $C$ is assumed to be a bounded demicontinuous operator satisfying condition $\left(S_{+}\right)$. For this, a key tool is the Browder degree given in [6]. Next, we consider two types of the operator $C$ for eigenvalue problem (E). For the one, we apply the Browder degree theory for nonlinear operators of the form $T+f$ with $T$ maximal monotone and $f$ bounded with condition $\left(S_{+}\right)$introduced in [7]. In the other case, where $C$ is a generalized pseudomonotone, quasibounded, and densely defined operator, we solve this problem by using the Kartsatos-Skrypnik degree theory for densely defined $\left(\tilde{S}_{+}\right)$-perturbations of maximal monotone operators developed in [8].

This paper is organized as follows. In Section 2, we study the solvability of implicit eigenvalue problem (IE) with normalized conditions based on the Browder degree theory. Section 3 contains an eigenvalue result for problem (E) as a special case of (IE). In Section 4, we deal with eigenvalue problem (E) for densely defined perturbations of maximal monotone operators under normalized conditions.

Let $X$ be a real Banach space with dual space $X^{*}, \Omega$ be a nonempty subset of $X$, and $Y$ be another real Banach space. Let $\bar{\Omega}$, int $\Omega$, and $\partial \Omega$ denote the closure, the interior, and the boundary of $\Omega$ in $X$, respectively. The symbol $\rightarrow(\rightarrow)$ stands for strong (weak) convergence. An operator $F: \Omega \rightarrow Y$ is said to be bounded if $F$ maps bounded subsets of $\Omega$ into bounded subsets of $Y$.F is said to be demicontinuous if for every $x_{0} \in \Omega$ and for every sequence $\left\{x_{n}\right\}$ in $\Omega$ with $x_{n} \rightarrow x_{0}$, we have $F x_{n} \rightarrow F x_{0}$.

Let $T: D(T) \subset X \rightarrow X^{*}$ be an operator. Then $T$ is said to be monotone if

$$
\langle T x-T y, x-y\rangle \geq 0 \quad \text { for every } x, y \in D(T) .
$$

$T$ is said to be maximal monotone if it is monotone and it follows from $\left(x, x^{*}\right) \in X \times X^{*}$ and

$$
\left\langle x^{*}-T y, x-y\right\rangle \geq 0 \quad \text { for every } y \in D(T)
$$

that $x \in D(T)$ and $T x=x^{*}$.

$T$ is said to be generalized pseudomonotone if for every sequence $\left\{x_{n}\right\}$ in $D(T)$ with $x_{n} \rightarrow$ $x_{0}, T x_{n} \rightarrow h^{*}$ and

$$
\limsup _{n \rightarrow \infty}\left\langle T x_{n}, x_{n}-x_{0}\right\rangle \leq 0,
$$

we have $x_{0} \in D(T), T x_{0}=h^{*}$, and $\lim _{n \rightarrow \infty}\left\langle T x_{n}, x_{n}\right\rangle=\left\langle T x_{0}, x_{0}\right\rangle$. 
We say that $T$ satisfies condition $\left(\tilde{S}_{+}\right)$if for every sequence $\left\{x_{n}\right\}$ in $D(T)$ with $x_{n} \rightarrow x_{0}$, $T x_{n} \rightarrow h^{*}$ and

$$
\limsup _{n \rightarrow \infty}\left\langle T x_{n}, x_{n}-x_{0}\right\rangle \leq 0
$$

we have $x_{n} \rightarrow x_{0}, x_{0} \in D(T)$, and $T x_{0}=h^{*}$.

We say that $T$ satisfies condition $\left(S_{+}\right)$if for every sequence $\left\{x_{n}\right\}$ in $D(T)$ with $x_{n} \rightarrow x_{0}$ and

$$
\limsup _{n \rightarrow \infty}\left\langle T x_{n}, x_{n}-x_{0}\right\rangle \leq 0,
$$

we have $x_{n} \rightarrow x_{0}$.

We say that $T$ satisfies condition $\left(S_{0}\right)$ on a set $M \subset D(T)$ if for every sequence $\left\{x_{n}\right\}$ in $M$ with $x_{n} \rightarrow x_{0}$ and $T x_{n} \rightarrow h^{*}$ and

$$
\lim _{n \rightarrow \infty}\left\langle T x_{n}, x_{n}\right\rangle=\left\langle h^{*}, x_{0}\right\rangle
$$

we have $x_{n} \rightarrow x_{0}$.

We say that $T$ satisfies condition $\left(S_{q}\right)$ on a set $M \subset D(T)$ if for every sequence $\left\{x_{n}\right\}$ in $M$ with $x_{n} \rightarrow x_{0}$ and $T x_{n} \rightarrow h^{*}$, we have $x_{n} \rightarrow x_{0}$.

We say that $T$ satisfies condition $\left(T_{\infty}^{(0)}\right)$ on a set $M \subset D(T)$ if for every sequence $\left\{x_{n}\right\}$ in $M$ with $\left\|T x_{n}\right\| \rightarrow \infty$, we have $\left\|T x_{n}\right\|^{-1} T x_{n} \rightarrow 0$.

It is obvious from the definitions that $\left(S_{+}\right)$implies $\left(S_{0}\right)$ and $\left(S_{0}\right)$ implies $\left(S_{q}\right)$. Note that if $T$ satisfies condition $\left(\tilde{S}_{+}\right)$and $X$ is reflexive, then $T$ is generalized pseudomonotone. For the above definitions, we refer to, e.g., [3, 4, 8], and [5].

Let $C:[0, \infty) \times M \rightarrow X^{\prime \prime}$ be an operator, where $M$ is a subset of $X$. Then $C(t, x)$ is said to be continuous in $t$ uniformly with respect to $x \in M$ if for every $t_{0} \in[0, \infty)$ and for every sequence $\left\{t_{n}\right\}$ in $[0, \infty)$ with $t_{n} \rightarrow t_{0}$, we have $C\left(t_{n}, x\right) \rightarrow C\left(t_{0}, x\right)$ uniformly with respect to $x \in M$.

We say that $C$ satisfies condition $\left(S_{+}\right)$if for every $\lambda \in(0, \infty)$ and for every sequence $\left\{x_{n}\right\}$ in $M$ with $x_{n} \rightarrow x_{0}$ and

$$
\limsup _{n \rightarrow \infty}\left\langle C\left(\lambda, x_{n}\right), x_{n}-x_{0}\right\rangle \leq 0,
$$

we have $x_{n} \rightarrow x_{0}$.

Throughout this paper, $X$ will always be an infinite dimensional real reflexive Banach space which has been renormed so that $X$ and $X^{\prime \prime}$ are locally uniformly convex.

An operator $J_{\psi}: X \rightarrow X^{\prime \prime}$ is said to be a duality operator if

$$
\left\langle J_{\psi} x, x\right\rangle=\psi(\|x\|)\|x\| \quad \text { and } \quad\left\|J_{\psi} x\right\|=\psi(\|x\|) \quad \text { for } x \in X \text {, }
$$

where $\psi:[0, \infty) \rightarrow[0, \infty)$ is continuous, strictly increasing, $\psi(0)=0$, and $\psi(t) \rightarrow \infty$ as $t \rightarrow \infty$ called a gauge function. If $\psi$ is the identity map $I$, then $J:=J_{I}$ is called a normalized duality operator. It is known in [14] that $J_{\psi}$ is continuous, bounded, surjective, strictly monotone, maximal monotone and satisfies condition $\left(S_{+}\right)$.

The following demiclosedness property of maximal monotone operators will be frequently used; see [5]. 
Lemma 1.1 Let $T: D(T) \subset X \rightarrow X^{*}$ be a maximal monotone operator. Then for every sequence $\left\{x_{n}\right\}$ in $D(T), x_{n} \rightarrow x$ in $X$ and $T x_{n} \rightarrow x^{*}$ in $X^{*}$ imply that $x \in D(T)$ and $T x=x^{*}$.

\section{Implicit eigenvalue problem}

In this section, we establish the existence of a solution for a nonlinear implicit eigenvalue problem under normalized conditions by using the Browder degree theory for class $\left(S_{+}\right)$.

Recall that a mapping $H:[0,1] \times \bar{\Omega} \rightarrow X^{*}$ is of class $\left(S_{+}\right)$if the following condition holds: For any sequence $\left\{u_{j}\right\}$ in $\bar{\Omega}$ with $u_{j} \rightarrow u_{0}$ and any sequence $\left\{t_{j}\right\}$ in $[0,1]$ with $t_{j} \rightarrow t_{0}$ for which

$$
\limsup _{j \rightarrow \infty}\left\langle H\left(t_{j}, u_{j}\right), u_{j}-u_{0}\right\rangle \leq 0
$$

we have $u_{j} \rightarrow u_{0}$ and $H\left(t_{j}, u_{j}\right) \rightarrow H\left(t_{0}, u_{0}\right)$; see $[2,6]$.

As mentioned in the introduction, Kartsatos and Skrypnik [4] gave the following result provided that property $(\mathcal{P})$ is fulfilled. In a more concrete situation, we adopt the normalization method considered in [3].

Theorem 2.1 Let $\Omega$ be a bounded open set in $X$ with $0 \in \Omega$. Let $T: D(T) \subset X \rightarrow X^{*}$ be a maximal monotone operator such that the closure of $\Omega$ is included in the interior of $D(T)$ with $T(0)=0$ and $T$ satisfies condition $\left(T_{\infty}^{(0)}\right)$ on $\bar{\Omega}$. Assume that $C:[0, \infty) \times \bar{\Omega} \rightarrow X^{*}$ is demicontinuous, bounded and satisfies condition $\left(S_{+}\right)$such that $C(0, x)=0$ for all $x \in \bar{\Omega}$ and $C(t, x)$ is continuous in $t$ uniformly with respect to $x \in \bar{\Omega}$. Further assume that

(c1) There exists a positive number $\mathcal{N}$ such that the weak sequential closure of the set

$$
G=\left\{\frac{C(\lambda, x)}{\|C(\lambda, x)\|}: \lambda \geq \mathcal{N}, x \in \bar{\Omega},\|J x+T x\| \leq 2 M(\lambda)\right\}
$$

does not contain the origin 0 , where

$$
M(\lambda)=\sup \{\|C(\lambda, x)\|: x \in \bar{\Omega}\}
$$

(c2) $\lim _{\lambda \rightarrow \infty} m(\lambda)=\infty$, where $m(\lambda)=\inf \{\|C(\lambda, x)\|: x \in \bar{\Omega}\}$.

Then the following statements hold:

(a) For each $\varepsilon>0$, there exists a point $\left(\lambda_{\varepsilon}, x_{\varepsilon}\right)$ in $(0, \infty) \times \partial \Omega$ such that

$$
T x_{\varepsilon}+C\left(\lambda_{\varepsilon}, x_{\varepsilon}\right)+\varepsilon J x_{\varepsilon}=0
$$

(b) If $0 \notin T(\partial \Omega)$ and $T$ satisfies condition $\left(S_{q}\right)$ on $\partial \Omega$, then the implicit eigenvalue problem

$$
T x+C(\lambda, x)=0
$$

has a solution $\left(\lambda_{0}, x_{0}\right)$ in $(0, \infty) \times \partial \Omega$.

Proof (a) For our aim, we use the Browder degree $d_{B}$ given in [6]. Let $\varepsilon$ be any positive number. We first prove that there is a number $\Lambda \in(0, \infty)$ such that

$$
d_{B}(T+C(\Lambda, \cdot)+\varepsilon J, \Omega, 0)=0 .
$$


Assume the contrary. For a sequence $\left\{\Lambda_{n}\right\}$ in $(0, \infty)$ with $\Lambda_{n} \rightarrow \infty$, the following occurs:

For each $n \in \mathbb{N}$, either there exists a point $x_{n} \in \Omega$ such that $T x_{n}+C\left(\Lambda_{n}, x_{n}\right)+\varepsilon J x_{n}=0$, in view of $d_{B}\left(T+C\left(\Lambda_{n}, \cdot\right)+\varepsilon J, \Omega, 0\right) \neq 0$, or there exists a point $x_{n} \in \partial \Omega$ such that $T x_{n}+$ $C\left(\Lambda_{n}, x_{n}\right)+\varepsilon J x_{n}=0$. Thus, we get a sequence $\left\{x_{n}\right\}$ in $\bar{\Omega}$ such that

$$
T x_{n}+C\left(\Lambda_{n}, x_{n}\right)+\varepsilon J x_{n}=0 .
$$

This implies

$$
\left\|J x_{n}+T x_{n}\right\| \leq\left\|(1-\varepsilon) J x_{n}\right\|+\left\|C\left(\Lambda_{n}, x_{n}\right)\right\| \leq 2 M\left(\Lambda_{n}\right)
$$

for sufficiently large $n$. Since the sequence $\left\{\left\|C\left(\Lambda_{n}, x_{n}\right)\right\|^{-1} C\left(\Lambda_{n}, x_{n}\right)\right\}$ is bounded in the reflexive Banach space $X^{*}$, we may suppose that $\left\|C\left(\Lambda_{n}, x_{n}\right)\right\|^{-1} C\left(\Lambda_{n}, x_{n}\right)$ converges weakly to some $h_{0} \in X^{*}$. Using (c1), it is clear that $h_{0} \neq 0$. It follows from (2.2) that

$$
\frac{T x_{n}}{\left\|T x_{n}\right\|} \rightarrow-h_{0}
$$

However, $\left\|C\left(\Lambda_{n}, x_{n}\right)\right\| \rightarrow \infty$ by (c2) implies $\left\|T x_{n}\right\| \rightarrow \infty$, which is a contradiction to condition $\left(T_{\infty}^{(0)}\right)$. Hence assertion (2.1) holds.

Next, we consider a mapping $H:[0,1] \times \bar{\Omega} \rightarrow X^{*}$ given by

$$
H(t, x)=T x+C(t \Lambda, x)+\varepsilon J x \quad \text { for }(t, x) \in[0,1] \times \bar{\Omega} .
$$

Then $H$ is of class $\left(S_{+}\right)$. To prove this, let $\left\{u_{j}\right\}$ be any sequence in $\bar{\Omega}$ with $u_{j} \rightarrow u_{0}$ and $\left\{t_{j}\right\}$ be any sequence in $[0,1]$ with $t_{j} \rightarrow t_{0}$ such that

$$
\limsup _{j \rightarrow \infty}\left\langle H\left(t_{j}, u_{j}\right), u_{j}-u_{0}\right\rangle \leq 0
$$

Since the operators $T$ and $J$ are monotone, it follows from

$$
\left\langle H\left(t_{j}, u_{j}\right), u_{j}-u_{0}\right\rangle=\left\langle T u_{j}, u_{j}-u_{0}\right\rangle+\left\langle C\left(t_{j} \Lambda, u_{j}\right), u_{j}-u_{0}\right\rangle+\varepsilon\left\langle J u_{j}, u_{j}-u_{0}\right\rangle
$$

that

$$
\left\langle H\left(t_{j}, u_{j}\right), u_{j}-u_{0}\right\rangle \geq\left\langle T u_{0}, u_{j}-u_{0}\right\rangle+\left\langle C\left(t_{j} \Lambda, u_{j}\right), u_{j}-u_{0}\right\rangle+\varepsilon\left\langle J u_{0}, u_{j}-u_{0}\right\rangle .
$$

By (2.4) and (2.6), we have

$$
\limsup _{j \rightarrow \infty}\left\langle C\left(t_{j} \Lambda, u_{j}\right), u_{j}-u_{0}\right\rangle \leq 0
$$

There are two cases to consider. If $t_{0}=0$, then $C\left(t_{j} \Lambda, u_{j}\right) \rightarrow 0$ and so

$$
\lim _{j \rightarrow \infty}\left\langle C\left(t_{j} \Lambda, u_{j}\right), u_{j}-u_{0}\right\rangle=0
$$


Since (2.5) implies

$$
\left\langle H\left(t_{j}, u_{j}\right), u_{j}-u_{0}\right\rangle \geq\left\langle T u_{0}, u_{j}-u_{0}\right\rangle+\left\langle C\left(t_{j} \Lambda, u_{j}\right), u_{j}-u_{0}\right\rangle+\varepsilon\left\langle J u_{j}, u_{j}-u_{0}\right\rangle
$$

it follows from (2.4) and (2.8) that

$$
\limsup _{j \rightarrow \infty}\left\langle J u_{j}, u_{j}-u_{0}\right\rangle \leq 0
$$

Since $J$ satisfies condition $\left(S_{+}\right)$, we obtain

$$
u_{j} \rightarrow u_{0}
$$

which implies

$$
T u_{j} \rightarrow T u_{0}, \quad C\left(t_{j} \Lambda, u_{j}\right) \rightarrow C\left(0, u_{0}\right), \quad \text { and } J u_{j} \rightarrow J u_{0}
$$

on observing that $T$ is demicontinuous on $\bar{\Omega}$. This means that $H\left(t_{j}, u_{j}\right) \rightarrow H\left(0, u_{0}\right)$. If $t_{0}>$ 0 , we have

$$
\begin{aligned}
& \limsup _{j \rightarrow \infty}\left\langle C\left(t_{0} \Lambda, u_{j}\right), u_{j}-u_{0}\right\rangle \\
& \quad \leq \limsup _{j \rightarrow \infty}\left\langle C\left(t_{j} \Lambda, u_{j}\right), u_{j}-u_{0}\right\rangle+\limsup _{j \rightarrow \infty}\left[-\left\langle C\left(t_{j} \Lambda, u_{j}\right)-C\left(t_{0} \Lambda, u_{j}\right), u_{j}-u_{0}\right\rangle\right]
\end{aligned}
$$

and hence by (2.7),

$$
\limsup _{j \rightarrow \infty}\left\langle C\left(t_{0} \Lambda, u_{j}\right), u_{j}-u_{0}\right\rangle \leq \limsup _{j \rightarrow \infty}\left[\left\|C\left(t_{j} \Lambda, u_{j}\right)-C\left(t_{0} \Lambda, u_{j}\right)\right\|\left\|u_{j}-u_{0}\right\|\right]=0
$$

Since $C$ satisfies condition $\left(S_{+}\right)$, we get $u_{j} \rightarrow u_{0}$ from which $T u_{j} \rightarrow T u_{0}, C\left(t_{j} \Lambda, u_{j}\right) \rightarrow$ $C\left(t_{0} \Lambda, u_{0}\right)$, and $J u_{j} \rightarrow J u_{0}$. Consequently, $H\left(t_{j}, u_{j}\right) \rightarrow H\left(t_{0}, u_{0}\right)$. We have just shown that the mapping $H$ is of class $\left(S_{+}\right)$.

We are now ready to apply the degree theory of Browder [6, 7]. Then we have

$$
d_{B}(H(0, \cdot), \Omega, 0)=d_{B}(T+\varepsilon J, \Omega, 0)=1 .
$$

The last equality is based on Theorem 3 in [6] because the operator $T+\varepsilon J$ is strictly monotone and demicontinuous on $\bar{\Omega}$ and satisfies condition $\left(S_{+}\right)$. On the other hand, it is shown in (2.1) that

$$
d_{B}(H(1, \cdot), \Omega, 0)=d_{B}(T+C(\Lambda, \cdot)+\varepsilon J, \Omega, 0)=0 .
$$

Hence, in view of Theorem 4 in [6], there exist $t_{0} \in[0,1]$ and $x_{0} \in \partial \Omega$ such that

$$
T x_{0}+C\left(t_{0} \Lambda, x_{0}\right)+\varepsilon J x_{0}=0
$$

It follows from the injectivity of $T+\varepsilon J$ that $t_{0}>0$. Consequently, if we let $\lambda_{\varepsilon}:=t_{0} \Lambda$ and $x_{\varepsilon}:=x_{0}$, then we have $\lambda_{\varepsilon} \in(0, \infty)$ and $T x_{\varepsilon}+C\left(\lambda_{\varepsilon}, x_{\varepsilon}\right)+\varepsilon J x_{\varepsilon}=0$. 
(b) Let $\left\{\varepsilon_{n}\right\}$ be a sequence in $(0, \infty)$ such that $\varepsilon_{n} \rightarrow 0$. According to statement (a), there exists a sequence $\left\{\left(\lambda_{\varepsilon_{n}}, x_{\varepsilon_{n}}\right)\right\}$ in $(0, \infty) \times \partial \Omega$ such that

$$
T x_{\varepsilon_{n}}+C\left(\lambda_{\varepsilon_{n}}, x_{\varepsilon_{n}}\right)+\varepsilon_{n} J x_{\varepsilon_{n}}=0
$$

If we set $x_{n}:=x_{\varepsilon_{n}}$ and $\lambda_{n}:=\lambda_{\varepsilon_{n}}$, it can be written in the form

$$
T x_{n}+C\left(\lambda_{n}, x_{n}\right)+\varepsilon_{n} J x_{n}=0
$$

Without loss of generality, we may suppose that

$$
\lambda_{n} \rightarrow \lambda_{0}, \quad x_{n} \rightarrow x_{0}, \quad \text { and } \quad C\left(\lambda_{n}, x_{n}\right) \rightarrow c^{*},
$$

where $\lambda_{0} \in[0, \infty], x_{0} \in X$, and $c^{\prime \prime} \in X^{*}$. Note that $\lambda_{0}$ belongs to $(0, \infty)$. In fact, if $\lambda_{0}=0$, then $C\left(\lambda_{n}, x_{n}\right) \rightarrow 0$ implies $T x_{n} \rightarrow 0$. Since $T$ satisfies condition $\left(S_{q}\right)$ on $\partial \Omega$, we obtain that $x_{n} \rightarrow x_{0} \in \partial \Omega$ and therefore $T x_{0}=0$, which contradicts the hypothesis that $0 \notin T(\partial \Omega)$. If $\lambda_{0}=\infty$, then (c2) implies $\left\|C\left(\lambda_{n}, x_{n}\right)\right\| \rightarrow \infty$ and so $\left\|T x_{n}\right\| \rightarrow \infty$. As in (2.3), a similar argument proves that $\left\|T x_{n}\right\|^{-1} T x_{n}$ converges weakly to some nonzero vector, which contradicts condition $\left(T_{\infty}^{(0)}\right)$. Thus we have shown that $\lambda_{0} \in(0, \infty)$.

For the next aim, we now show that

$$
\limsup _{n \rightarrow \infty}\left\langle C\left(\lambda_{n}, x_{n}\right), x_{n}-x_{0}\right\rangle \leq 0
$$

Assume that (2.11) is false. Then there exists a subsequence of $\left\{x_{n}\right\}$, denoted again by $\left\{x_{n}\right\}$, such that

$$
\lim _{n \rightarrow \infty}\left\langle C\left(\lambda_{n}, x_{n}\right), x_{n}-x_{0}\right\rangle>0
$$

Hence we obtain from (2.9) that

$$
\limsup _{n \rightarrow \infty}\left\langle T x_{n}, x_{n}-x_{0}\right\rangle<0
$$

Noticing by (2.9) and (2.10) that $T x_{n} \rightarrow-c^{*}$, we get

$$
\limsup _{n \rightarrow \infty}\left\langle T x_{n}, x_{n}\right\rangle<\lim _{n \rightarrow \infty}\left\langle T x_{n}, x_{0}\right\rangle=\left\langle-c^{*}, x_{0}\right\rangle
$$

For every $x \in D(T)$, we have by the monotonicity of $T$

$$
\begin{aligned}
\liminf _{n \rightarrow \infty}\left\langle T x_{n}, x_{n}\right\rangle & \geq \liminf _{n \rightarrow \infty}\left[\left\langle T x_{n}, x\right\rangle+\left\langle T x, x_{n}-x\right\rangle\right] \\
& =\left\langle-c^{*}, x\right\rangle+\left\langle T x, x_{0}-x\right\rangle,
\end{aligned}
$$

which implies along with (2.12)

$$
\left\langle-c^{*}-T x, x_{0}-x\right\rangle>0
$$

By the maximal monotonicity of $T$, we have $x_{0} \in D(T)$ and $T x_{0}=-c^{*}$. Letting $x=x_{0}$ in (2.13), we get a contradiction. Therefore, (2.11) is true. 
Since $C\left(\lambda_{n}, x_{n}\right)-C\left(\lambda_{0}, x_{n}\right) \rightarrow 0$, it follows from (2.11) and

$$
\left\langle C\left(\lambda_{n}, x_{n}\right), x_{n}-x_{0}\right\rangle=\left\langle C\left(\lambda_{n}, x_{n}\right)-C\left(\lambda_{0}, x_{n}\right), x_{n}-x_{0}\right\rangle+\left\langle C\left(\lambda_{0}, x_{n}\right), x_{n}-x_{0}\right\rangle
$$

that

$$
\limsup _{n \rightarrow \infty}\left\langle C\left(\lambda_{0}, x_{n}\right), x_{n}-x_{0}\right\rangle \leq 0
$$

Since $C$ satisfies condition $\left(S_{+}\right)$and $\lambda_{0} \in(0, \infty)$, we have $x_{n} \rightarrow x_{0} \in \partial \Omega$, which implies $C\left(\lambda_{n}, x_{n}\right) \rightarrow C\left(\lambda_{0}, x_{0}\right)$. Hence we obtain from (2.9) that $T x_{n} \rightarrow-C\left(\lambda_{0}, x_{0}\right)$. By Lemma 1.1, we conclude that $T x_{0}+C\left(\lambda_{0}, x_{0}\right)=0$. This completes the proof.

Remark 2.2 In the proof of Theorem 2.1, the demicontinuity of $T$ on $\bar{\Omega}$ is needed to show that $H$ is of class $\left(S_{+}\right)$. This is guaranteed under an additional condition $\bar{\Omega} \subset \operatorname{int} D(T)$. Actually, local boundedness of $T$ on int $D(T)$ implies the demicontinuity of $T$; see, e.g., [5].

\section{Eigenvalue problem with condition $\left(S_{+}\right)$}

In this section, we study a multiplicative eigenvalue problem as a special case of the implicit eigenvalue problem in the previous section. As a key tool, we employ the Browder degree for nonlinear operators of the form $T+f$ with $T$ maximal monotone and $f$ bounded with condition $\left(S_{+}\right)$.

We give a variant of Corollary 1 in [4] under normalized conditions.

Theorem 3.1 Let $\Omega$ be a bounded open set in $X$ with $0 \in \Omega$. Let $T: D(T) \subset X \rightarrow X^{*}$ be a maximal monotone operator with $0 \in D(T)$ and $T(0)=0$ such that $T$ satisfies condition $\left(T_{\infty}^{(0)}\right)$ on $D(T) \cap \bar{\Omega}$. Assume that $C: \bar{\Omega} \rightarrow X^{*}$ is a demicontinuous bounded operator which satisfies condition $\left(S_{+}\right)$and the two additional conditions:

(c1) There is a positive number $\mathcal{N}$ such that the weak sequential closure of the set

$$
G=\left\{\frac{C x}{\|C x\|}: \lambda \geq \mathcal{N}, x \in \bar{\Omega},\|J x+T x\| \leq 2 M(\lambda)\right\}
$$

does not contain zero vector, where

$$
M(\lambda)=|\lambda| \sup \{\|C x\|: x \in \bar{\Omega}\}
$$

(c2) $\inf \{\|C x\|: x \in \bar{\Omega}\}$ is not equal to 0.

Then we have the following properties:

(a) For each $\varepsilon>0$, there exists $\left(\lambda_{\varepsilon}, x_{\varepsilon}\right) \in(0, \infty) \times(D(T) \cap \partial \Omega)$ such that

$$
T x_{\varepsilon}+\lambda_{\varepsilon} C x_{\varepsilon}+\varepsilon J x_{\varepsilon}=0 \text {. }
$$

(b) If $0 \notin T(D(T) \cap \partial \Omega)$ and $T$ satisfies condition $\left(S_{q}\right)$ on $D(T) \cap \partial \Omega$, then the eigenvalue problem

$$
T x+\lambda C x=0
$$

has a solution $\left(\lambda_{0}, x_{0}\right)$ in $(0, \infty) \times(D(T) \cap \partial \Omega)$. 
Proof (a) Fix $\varepsilon>0$. Let $d_{B}$ denote the Browder degree in the sense of [7]. We first claim that there exists a number $\Lambda$ in $(0, \infty)$ such that

$$
d_{B}(T+\Lambda C+\varepsilon J, \Omega, 0)=0 .
$$

Assume the contrary. As in the proof of (2.1), a similar argument establishes that for a sequence $\left\{\Lambda_{n}\right\}$ in $(0, \infty)$ with $\Lambda_{n} \rightarrow \infty$, there is a sequence $\left\{x_{n}\right\}$ in $D(T) \cap \bar{\Omega}$ such that

$$
T x_{n}+\Lambda_{n} C x_{n}+\varepsilon J x_{n}=0
$$

This implies

$$
\left\|J x_{n}+T x_{n}\right\| \leq\left\|(1-\varepsilon) J x_{n}\right\|+\left\|\Lambda_{n} C x_{n}\right\| \leq 2 M\left(\Lambda_{n}\right)
$$

for sufficiently large $n$. By the boundedness of the sequence $\left\{\left\|C x_{n}\right\|^{-1} C x_{n}\right\}$ in $X^{*}$, we may suppose that $\left\|C x_{n}\right\|^{-1} C x_{n} \rightarrow h_{0}$ for some $h_{0} \in X^{*}$. It follows from (c1) and (3.2) that $h_{0} \neq 0$ and

$$
\frac{T x_{n}}{\left\|T x_{n}\right\|} \rightarrow-h_{0}
$$

But (c2) implies $\left\|T x_{n}\right\| \rightarrow \infty$, which contradicts condition $\left(T_{\infty}^{(0)}\right)$. Hence assertion (3.1) holds.

Now we consider a mapping $H:[0,1] \times(D(T) \cap \bar{\Omega}) \rightarrow X^{*}$ given by

$$
H(t, x):=T x+\varepsilon J x+t \Lambda C x \quad \text { for }(t, x) \in[0,1] \times(D(T) \cap \bar{\Omega}) .
$$

Using the normalization property of the Browder degree $d_{B}, e . g$., Theorem 12 in [7], we have

$$
d_{B}(H(0, \cdot), \Omega, 0)=d_{B}(T+\varepsilon J, \Omega, 0)=1 .
$$

Moreover, (3.1) means that

$$
d_{B}(H(1, \cdot), \Omega, 0)=d_{B}(T+\varepsilon J+\Lambda C, \Omega, 0)=0 .
$$

Note that $T+\varepsilon J$ is maximal monotone and satisfies condition $\left(S_{+}\right)$. Hence, there are $t_{0} \in$ $[0,1]$ and $x_{0} \in D(T) \cap \partial \Omega$ such that

$$
T x_{0}+t_{0} \Lambda C x_{0}+\varepsilon J x_{0}=0 .
$$

By the injectivity of the strictly monotone operator $T+\varepsilon J$, we know that $t_{0}>0$. Consequently, if we let $\lambda_{\varepsilon}:=t_{0} \Lambda$ and $x_{\varepsilon}:=x_{0}$, then $\lambda_{\varepsilon} \in(0, \infty)$ and $T x_{\varepsilon}+\lambda_{\varepsilon} C x_{\varepsilon}+\varepsilon J x_{\varepsilon}=0$.

(b) Let $\left\{\varepsilon_{n}\right\}$ be a sequence in $(0, \infty)$ such that $\varepsilon_{n} \rightarrow 0$. By (a), there exists a sequence $\left\{\left(\lambda_{n}, x_{n}\right)\right\}$ in $(0, \infty) \times(D(T) \cap \partial \Omega)$ such that

$$
T x_{n}+\lambda_{n} C x_{n}+\varepsilon_{n} J x_{n}=0 .
$$


We may suppose that

$$
\lambda_{n} \rightarrow \lambda_{0}, \quad x_{n} \rightarrow x_{0}, \quad \text { and } C x_{n} \rightarrow c^{*},
$$

where $\lambda_{0} \in[0, \infty], x_{0} \in X$, and $c^{*} \in X^{*}$. Note that $\lambda_{0}$ belongs to $(0, \infty)$. Indeed, if $\lambda_{0}=0$, then it follows from $T x_{n} \rightarrow 0$ and condition $\left(S_{q}\right)$ that $x_{n} \rightarrow x_{0} \in \partial \Omega$ and therefore $x_{0} \in$ $D(T)$ and $T x_{0}=0$, which contradicts the hypothesis that $0 \notin T(D(T) \cap \partial \Omega)$. If $\lambda_{0}=\infty$, then (c2) implies $\left\|\lambda_{n} C x_{n}\right\| \rightarrow \infty$ and so $\left\|T x_{n}\right\| \rightarrow \infty$. But we can show as above that $\left\|T x_{n}\right\|^{-1} T x_{n}$ converges weakly to some nonzero vector, which contradicts condition $\left(T_{\infty}^{(0)}\right)$.

To prove that

$$
\limsup _{n \rightarrow \infty}\left\langle\lambda_{n} C x_{n}, x_{n}-x_{0}\right\rangle \leq 0,
$$

we assume to the contrary that there exists a subsequence of $\left\{x_{n}\right\}$, denoted again by $\left\{x_{n}\right\}$, such that

$$
\lim _{n \rightarrow \infty}\left\langle\lambda_{n} C x_{n}, x_{n}-x_{0}\right\rangle>0
$$

Hence we obtain from (3.3) that

$$
\limsup _{n \rightarrow \infty}\left\langle T x_{n}, x_{n}-x_{0}\right\rangle<0
$$

Noticing by (3.3) and (3.4) that $T x_{n} \rightarrow-\lambda_{0} c^{*}$, we get

$$
\limsup _{n \rightarrow \infty}\left\langle T x_{n}, x_{n}\right\rangle<\lim _{n \rightarrow \infty}\left\langle T x_{n}, x_{0}\right\rangle=\left\langle-\lambda_{0} c^{*}, x_{0}\right\rangle
$$

For every $x \in D(T)$, we have by the monotonicity of $T$

$$
\liminf _{n \rightarrow \infty}\left\langle T x_{n}, x_{n}\right\rangle \geq\left\langle-\lambda_{0} c^{\prime \prime}, x\right\rangle+\left\langle T x, x_{0}-x\right\rangle,
$$

which implies along with (3.6)

$$
\left\langle-\lambda_{0} c^{*}-T x, x_{0}-x\right\rangle>0
$$

By the maximal monotonicity of $T$, we get a contradiction. Therefore, (3.5) holds.

It follows from (3.5) and

$$
\left\langle\lambda_{n} C x_{n}, x_{n}-x_{0}\right\rangle=\left(\lambda_{n}-\lambda_{0}\right)\left\langle C x_{n}, x_{n}-x_{0}\right\rangle+\left\langle\lambda_{0} C x_{n}, x_{n}-x_{0}\right\rangle
$$

that

$$
\lambda_{0} \limsup _{n \rightarrow \infty}\left\langle C x_{n}, x_{n}-x_{0}\right\rangle \leq 0 .
$$

Since $C$ satisfies condition $\left(S_{+}\right)$and is demicontinuous, we have $x_{n} \rightarrow x_{0} \in \partial \Omega$, which implies $C x_{n} \rightarrow C x_{0}$. Hence we obtain from (3.3) that $T x_{n}--\lambda_{0} C x_{0}$. By Lemma 1.1, we conclude that $x_{0} \in D(T) \cap \partial \Omega$ and $T x_{0}+\lambda_{0} C x_{0}=0$, what we wanted to prove. 
Remark 3.2 We point out that in Theorem 3.1, the condition $\bar{\Omega} \subset \operatorname{int} D(T)$ is not necessary to be assumed.

\section{Densely defined perturbations}

This section is devoted to the eigenvalue problem for densely defined quasibounded perturbations of maximal monotone operators in reflexive Banach spaces. To do this, we apply the Kartsatos-Skrypnik degree for densely defined $\left(\tilde{S}_{+}\right)$-perturbations of maximal monotone operators developed in [8].

Recall that an operator $C: D(C) \subset X \rightarrow X^{*}$ is quasibounded if for every $S>0$ there exists a constant $K(S)>0$ such that for all $u \in D(C)$ with $\|u\| \leq S$ and $\langle C u, u\rangle \leq 0$, we have $\|C u\| \leq K(S)$.

As in Section 3, we employ a normalization method to obtain an eigenvalue result for generalized pseudomonotone operators.

Theorem 4.1 Let $\Omega$ be a bounded open set in $X$ with $0 \in \Omega$ and $L$ be a dense subspace of $X$. Let $T: D(T) \subset X \rightarrow X^{*}$ be a maximal monotone operator with $0 \in D(T)$ and $T(0)=0$ which satisfies condition $\left(T_{\infty}^{(0)}\right)$ on $D(T) \cap \bar{\Omega}$. Assume that $C: D(C) \subset X \rightarrow X^{*}$ is a generalized pseudomonotone quasibounded operator with $L \subset D(C)$. Furthermore, assume that

(h1) There exists a positive number $\mathcal{N}$ such that the weak sequential closure of the set

$$
G=\left\{\frac{C x}{\|C x\|}: \lambda \geq \mathcal{N}, x \in D(C) \cap \bar{\Omega},\left\|J_{\psi} x+T x\right\| \leq 2 M(\lambda)\right\}
$$

does not contain zero vector, where

$$
M(\lambda)=|\lambda| \sup \{\|C x\|: x \in D(C) \cap \bar{\Omega}\} .
$$

(h2) $\inf \{\|C x\|: x \in D(C) \cap \bar{\Omega}\}$ is not equal to 0 .

(h3) For every $F \in \mathcal{F}(L)$ and $v \in L$, the function $c(F, v): F \rightarrow \mathbb{R}, c(F, v)(u)=\langle C u, v\rangle$, is continuous on $F$, where $\mathcal{F}(L)$ denotes the set of all finite-dimensional subspaces of $L$. Then the following statements hold:

(a) For each $\varepsilon>0$, there exists a point $\left(\lambda_{\varepsilon}, x_{\varepsilon}\right)$ in $(0, \infty) \times(D(T+C) \cap \partial \Omega)$ such that

$$
T x_{\varepsilon}+\lambda_{\varepsilon} C x_{\varepsilon}+\varepsilon J_{\psi} x_{\varepsilon}=0
$$

Here $D(T+C)$ denotes the intersection of $D(T)$ and $D(C)$.

(b) If $0 \notin T\left(D(T) \cap \partial \Omega\right.$ ) and $T$ satisfies condition $\left(S_{0}\right)$ on $D(T) \cap \partial \Omega$, then the eigenvalue problem

$$
T x+\lambda C x=0
$$

has a solution $\left(\lambda_{0}, x_{0}\right)$ in $(0, \infty) \times(D(T+C) \cap \partial \Omega)$.

Proof (a) We will apply the Kartsatos-Skrypnik degree $d_{S}$ given in [8]. Let $\varepsilon$ be an arbitrary positive number. Since $T$ satisfies condition $\left(T_{\infty}^{(0)}\right)$ on $D(T) \cap \bar{\Omega}$ and $J_{\psi}$ is bounded, we can prove as in Theorem 3.1 that under hypotheses (h1) and (h2), there is a positive number 
$\Lambda$ such that

$$
d_{S}\left(T+\Lambda C+\varepsilon J_{\psi}, \Omega, 0\right)=0 .
$$

For $t \in[0,1]$, we set $T^{t}:=T$ and $C^{t}:=t \Lambda C+\varepsilon J_{\psi}$, where $D\left(T^{t}\right)$ and $D\left(C^{t}\right)$ denote the domain of $T^{t}$ and $C^{t}$, respectively. In this case, $D\left(T^{t}\right)=D(T)$ for $t \in[0,1], D\left(C^{0}\right)=X$ for $t=0$ and $D\left(C^{t}\right)=D(C)$ for $t \in(0,1]$. Notice that the operators $C^{0}=\varepsilon J_{\psi}$ and $C^{1}=\Lambda C+\varepsilon J_{\psi}$ satisfy condition $\left(\tilde{S}_{+}\right)$, based on the facts that $C$ is generalized pseudomonotone and $J_{\psi}$ is bounded and satisfies condition $\left(S_{+}\right)$.

In the sense of Definition 4.2 in [8], we check the following conditions on two families $\left\{T^{t}\right\}$ and $\left\{C^{t}\right\}$. In fact, conditions on $\left\{T^{t}\right\}$ are obviously satisfied, with $T^{t}$ independent of $t$, due to maximal monotonicity of $T, 0 \in D(T)$, and $T(0)=0$.

$\left(c_{1}^{t}\right)$ Since $J_{\psi}$ is monotone and bounded, it follows from the quasiboundedness of $C$ that $\left\{C^{t}\right\}$ is uniformly quasibounded.

$\left(c_{2}^{t}\right)$ Let $\left\{t_{n}\right\}$ be any sequence in $[0,1]$ and $\left\{u_{n}\right\}$ be any sequence in $L$ such that $t_{n} \rightarrow t_{0}$, $u_{n} \rightarrow u_{0}, C^{t_{n}} u_{n} \rightarrow h^{*}$ and

$$
\limsup _{n \rightarrow \infty}\left\langle C^{t_{n}} u_{n}, u_{n}-u_{0}\right\rangle \leq 0, \quad\left\langle C^{t_{n}} u_{n}, u_{n}\right\rangle \leq 0
$$

where $t_{0} \in[0,1], u_{0} \in X$, and $h^{*} \in X^{*}$. If $t_{0}=0$, then the second inequality in(4.2) implies

$$
\varepsilon \psi\left(\left\|u_{n}\right\|\right)\left\|u_{n}\right\|=\varepsilon\left\langle J_{\psi} u_{n}, u_{n}\right\rangle \leq-t_{n} \Lambda\left\langle C u_{n}, u_{n}\right\rangle \rightarrow 0
$$

and hence $u_{n} \rightarrow 0, u_{0}=0 \in D\left(C^{0}\right)$, and $C^{0} u_{0}=h^{*}$. Now let $t_{0}>0$. From the first inequality in (4.2) and the following inequality

$$
\left\langle C^{t_{n}} u_{n}, u_{n}-u_{0}\right\rangle \geq t_{n} \Lambda\left\langle C u_{n}, u_{n}-u_{0}\right\rangle+\varepsilon\left\langle J_{\psi} u_{0}, u_{n}-u_{0}\right\rangle
$$

we obtain that

$$
\limsup _{n \rightarrow \infty}\left\langle C u_{n}, u_{n}-u_{0}\right\rangle \leq 0
$$

In view of $C^{t_{n}} u_{n} \rightarrow h^{*}$, there exists a subsequence of $\left\{u_{n}\right\}$, denoted again by $\left\{u_{n}\right\}$, such that $C u_{n} \rightarrow h_{1}^{*}$ and $J_{\psi} u_{n} \rightarrow h_{2}^{*}$ for some $h_{1}^{*}, h_{2}^{*} \in X^{*}$. Since $C$ is generalized pseudomonotone, we obtain from (4.3) that $u_{0} \in D(C), C u_{0}=h_{1}^{*}$, and $\left\langle C u_{n}, u_{n}\right\rangle \rightarrow\left\langle C u_{0}, u_{0}\right\rangle$. Thus,

$$
\lim _{n \rightarrow \infty}\left\langle C u_{n}, u_{n}-u_{0}\right\rangle=\left\langle C u_{0}, u_{0}\right\rangle-\left\langle h_{1}^{*}, u_{0}\right\rangle=0
$$

Hence it follows from the first inequality in (4.2) that

$$
\varepsilon \limsup _{n \rightarrow \infty}\left\langle J_{\psi} u_{n}, u_{n}-u_{0}\right\rangle=\limsup _{n \rightarrow \infty}\left\langle t_{n} \Lambda C u_{n}+\varepsilon J_{\psi} u_{n}, u_{n}-u_{0}\right\rangle \leq 0
$$

Since $J_{\psi}$ satisfies condition $\left(S_{+}\right)$, we have $u_{n} \rightarrow u_{0}$ and so $J_{\psi} u_{n} \rightarrow J_{\psi} u_{0}$. Consequently, $u_{0} \in D\left(C^{t_{0}}\right)$ and $C^{t_{0}} u_{0}=t_{0} \Lambda h_{1}^{*}+\varepsilon h_{2}^{*}=h^{*}$. Therefore, condition $\left(c_{2}^{t}\right)$ is satisfied. 
$\left(c_{3}^{t}\right)$ For every $F \in \mathcal{F}(L)$ and $v \in L$, the function $\tilde{c}(F, v):[0,1] \times F \rightarrow \mathbb{R}, \tilde{c}(F, v)(t, u)=$ $\left\langle C^{t} u, v\right\rangle$, is continuous on $[0,1] \times F$ because $c(F, v)$ is continuous on $F$ and $J_{\psi}$ is continuous on $X$.

We can now consider a mapping $H:[0,1] \times(D(T+C) \cap \bar{\Omega}) \rightarrow X^{*}$ given by

$$
H(t, x):=T x+t \Lambda C x+\varepsilon J_{\psi} x .
$$

By Theorem 4.4 in [8] and (4.1), we have

$$
d_{S}(H(0, \cdot), \Omega, 0)=d_{S}\left(T+\varepsilon J_{\psi}, \Omega, 0\right)=1
$$

and

$$
d_{S}(H(1, \cdot), \Omega, 0)=d_{S}\left(T+\Lambda C+\varepsilon J_{\psi}, \Omega, 0\right)=0 .
$$

According to Theorem 4.3 in [8], there exist $t_{0} \in[0,1]$ and $x_{0} \in D(T+C) \cap \partial \Omega$ such that

$$
T x_{0}+t_{0} \Lambda C x_{0}+\varepsilon J_{\psi} x_{0}=0
$$

The injectivity of $T+\varepsilon J_{\psi}$ implies that $t_{0}>0$. If we let $\lambda_{\varepsilon}:=t_{0} \Lambda$ and $x_{\varepsilon}:=x_{0}$, then the conclusion follows.

(b) Let $\left\{\varepsilon_{n}\right\}$ be a sequence in $(0, \infty)$ such that $\varepsilon_{n} \rightarrow 0$. According to (a), there are sequences $\left\{\lambda_{n}\right\}$ in $(0, \infty)$ and $\left\{x_{n}\right\}$ in $D(T+C) \cap \partial \Omega$ such that

$$
T x_{n}+\lambda_{n} C x_{n}+\varepsilon_{n} J_{\psi} x_{n}=0
$$

Then it follows from the monotonicity of $T$ with $T(0)=0$ that

$$
\left\langle C x_{n}, x_{n}\right\rangle \leq-\frac{\varepsilon_{n}}{\lambda_{n}} \psi\left(\left\|x_{n}\right\|\right)\left\|x_{n}\right\| \leq 0
$$

Hence the quasiboundedness of $C$ implies that $\left\{C x_{n}\right\}$ is bounded. Without loss of generality, we may suppose that

$$
\lambda_{n} \rightarrow \lambda_{0}, \quad x_{n} \rightarrow x_{0}, \quad \text { and } \quad C x_{n} \rightarrow c^{*},
$$

where $\lambda_{0} \in[0, \infty], x_{0} \in X$, and $c^{*} \in X^{*}$. Since T satisfies conditions $\left(S_{q}\right)$ and $\left(T_{\infty}^{(0)}\right)$ and since $0 \notin T(D(T) \cap \partial \Omega)$, it is easily verified that $\lambda_{0}$ belongs to $(0, \infty)$.

As in the proof of Theorem 3.1, we can show that

$$
\limsup _{n \rightarrow \infty}\left\langle\lambda_{n} C x_{n}, x_{n}-x_{0}\right\rangle \leq 0 .
$$

Then it follows from (4.6) and $\lambda_{n} \rightarrow \lambda_{0}$ that

$$
\limsup _{n \rightarrow \infty}\left\langle C x_{n}, x_{n}-x_{0}\right\rangle \leq 0
$$


Since $C$ is generalized pseudomonotone, we have by (4.5)

$$
x_{0} \in D(C), \quad C x_{0}=c^{*}, \quad \text { and } \quad \lim _{n \rightarrow \infty}\left\langle C x_{n}, x_{n}\right\rangle=\left\langle C x_{0}, x_{0}\right\rangle .
$$

Hence we obtain from (4.4) that $T x_{n} \rightarrow-\lambda_{0} C x_{0}$ and

$$
\lim _{n \rightarrow \infty}\left\langle T x_{n}, x_{n}\right\rangle=\lim _{n \rightarrow \infty}-\lambda_{n}\left\langle C x_{n}, x_{n}\right\rangle=\left\langle-\lambda_{0} C x_{0}, x_{0}\right\rangle .
$$

Since $T$ satisfies condition $\left(S_{0}\right)$ on $D(T) \cap \partial \Omega$, we have $x_{n} \rightarrow x_{0}$. By the maximal monotonicity of $T$, we conclude that $x_{0} \in D(T+C) \cap \partial \Omega$ and $T x_{0}+\lambda_{0} C x_{0}=0$. This completes the proof.

As a consequence of Theorem 4.1, we get another eigenvalue result for densely defined operators satisfying condition $\left(\tilde{S}_{+}\right)$in comparison with Theorem 4 in [4].

Corollary 4.2 Let $T, \Omega$, and $L$ be as in Theorem 4.1. Assume that $C: D(C) \subset X \rightarrow X^{*}$ is a quasibounded operator with $L \subset D(C)$ and satisfies condition $\left(\tilde{S}_{+}\right)$. Furthermore, assume that conditions (h1), (h2), and (h3) in Theorem 4.1 are satisfied. Then:

(a) For each $\varepsilon>0$, there exists $\left(\lambda_{\varepsilon}, x_{\varepsilon}\right) \in(0, \infty) \times(D(T+C) \cap \partial \Omega)$ such that $T x_{\varepsilon}+\lambda_{\varepsilon} C x_{\varepsilon}+\varepsilon J_{\psi} x_{\varepsilon}=0$.

(b) If $0 \notin T(D(T) \cap \partial \Omega)$ and $T$ satisfies condition $\left(S_{q}\right)$ on $D(T) \cap \partial \Omega$, then there exists $\left(\lambda_{0}, x_{0}\right) \in(0, \infty) \times(D(T+C) \cap \partial \Omega)$ such that $T x_{0}+\lambda_{0} C x_{0}=0$.

Proof Statement (a) follows from part (a) of Theorem 4.1 by noting that if $C$ satisfies condition $\left(\tilde{S}_{+}\right)$, then $C$ is generalized pseudomonotone.

(b) Let $\left\{\varepsilon_{n}\right\}$ be a sequence in $(0, \infty)$ such that $\varepsilon_{n} \rightarrow 0$. In view of (a), we can choose a sequence $\left\{\left(\lambda_{n}, x_{n}\right)\right\}$ in $(0, \infty) \times(D(T+C) \cap \partial \Omega)$ such that

$$
T x_{n}+\lambda_{n} C x_{n}+\varepsilon_{n} J_{\psi} x_{n}=0 .
$$

We may suppose that

$$
\lambda_{n} \rightarrow \lambda_{0}, \quad x_{n} \rightarrow x_{0}, \quad \text { and } C x_{n} \rightarrow c^{*},
$$

where $\lambda_{0} \in[0, \infty], x_{0} \in X$, and $c^{*} \in X^{*}$. Obviously, $\lambda_{0} \in(0, \infty)$. As before, the same argument shows that

$$
\limsup _{n \rightarrow \infty}\left\langle C x_{n}, x_{n}-x_{0}\right\rangle \leq 0
$$

Since $C$ satisfies condition $\left(\tilde{S}_{+}\right)$, we have

$$
x_{n} \rightarrow x_{0}, \quad x_{0} \in D(C), \text { and } C x_{0}=c^{*} .
$$

Combining this with $T x_{n} \rightarrow-\lambda_{0} C x_{0}$, we obtain that $x_{0} \in D(T+C) \cap \partial \Omega$ and $T x_{0}+\lambda_{0} C x_{0}=$ 0 as required.

Remark 4.3 When $C$ satisfies condition $\left(\tilde{S}_{+}\right)$, condition $\left(S_{0}\right)$ on $T$ appearing in Theorem 4.1 may be replaced by weaker condition $\left(S_{q}\right)$; see the proof of Theorem 4.1. 
Competing interests

The authors declare that they have no competing interests.

\section{Authors' contributions}

BJ participated in the sequence alignment and coordination. KI conceived of the study and drafted the manuscript. All authors read and approved the final manuscript.

\section{Acknowledgements}

The authors would like to thank anonymous referees for valuable comments and suggestions. This research was supported by the Basic Science Research Program through the National Research Foundation of Korea (NRF) funded by the Ministry of Education, Science and Technology (NRF-2011-0021-829).

Received: 19 September 2012 Accepted: 9 February 2013 Published: 28 February 2013

\section{References}

1. Krasnosel'skii, MA: Topological Methods in the Theory of Nonlinear Integral Equations. Pergamon, New York (1964)

2. Browder, FE: Nonlinear operators and nonlinear equations of evolution in Banach spaces. In: Nonlinear Functional Analysis (Proc. Sympos. Pure Math., vol. XVIII, Part 2, Chicago, III., 1968), pp. 1-308. Am. Math. Soc., Providence (1976)

3. Kartsatos, AG, Skrypnik, IV: Normalized eigenvectors for nonlinear abstract and elliptic operators. J. Differ. Equ. 155, 443-475 (1999)

4. Kartsatos, AG, Skrypnik, IV: On the eigenvalue problem for perturbed nonlinear maximal monotone operators in reflexive Banach spaces. Trans. Am. Math. Soc. 358, 3851-3881 (2006)

5. Zeidler, E: Nonlinear Functional Analysis and Its Applications II/B: Nonlinear Monotone Operators. Springer, New York (1990)

6. Browder, FE: Degree of mapping for nonlinear mappings of monotone type. Proc. Natl. Acad. Sci. USA 80, 1771-1773 (1983)

7. Browder, FE: Fixed point theory and nonlinear problems. Bull. Am. Math. Soc. 9, 1-39 (1983)

8. Kartsatos, AG, Skrypnik, IV: A new topological degree theory for densely defined quasibounded $\left(\tilde{S}_{+}\right)$-perturbations of multivalued maximal monotone operators in reflexive Banach spaces. Abstr. Appl. Anal. 2005, 121-158 (2005)

9. Skrypnik, IV: Nonlinear Higher Order Elliptic Equations. Naukova Dumka, Kiev (Russian) (1973)

10. Skrypnik, IV: Methods for Analysis of Nonlinear Elliptic Boundary Value Problems. Transl., Ser. II., vol. 139. Am. Math. Soc., Providence (1994)

11. Guan, Z, Kartsatos, AG: On the eigenvalue problem for perturbations of nonlinear accretive and monotone operators in Banach spaces. Nonlinear Anal. 27, 125-141 (1996)

12. Kartsatos, AG: New results in the perturbation theory of maximal monotone and $m$-accretive operators in Banach spaces. Trans. Am. Math. Soc. 348, 1663-1707 (1996)

13. Li, H-X, Huang, F-L: On the nonlinear eigenvalue problem for perturbations of monotone and accretive operators in Banach spaces. Sichuan Daxue Xuebao 37, 303-309 (2000)

14. Petryshyn, WV: Approximation-Solvability of Nonlinear Functional and Differential Equations. Marcel Dekker, New York (1993)

doi:10.1186/1029-242X-2013-72

Cite this article as: Kim and Bae: Some eigenvalue results for perturbations of maximal monotone operators. Journal of Inequalities and Applications 2013 2013:72.

\section{Submit your manuscript to a SpringerOpen ${ }^{\circ}$ journal and benefit from:}

- Convenient online submission

Rigorous peer review

- Immediate publication on acceptance

- Open access: articles freely available online

- High visibility within the field

- Retaining the copyright to your article 